Mathematical Modelling and Analysis

Volume 20 Number 1, January 2015, 124-138

http://dx.doi.org/10.3846/13926292.2015.1006155

(c) Vilnius Gediminas Technical University, 2015
Publisher: Taylor\&Francis and VGTU

http://www.tandfonline.com/TMMA

ISSN: $1392-6292$

eISSN: $1648-3510$

\title{
Convergence of Comonotone Histopolating Splines
}

\section{Helle Hallik and Peeter Oja}

\author{
University of Tartu \\ J. Liivi 2, 50409 Tartu, Estonia \\ E-mail: Helle.Hallik@ut.ee \\ E-mail(corresp.): Peeter.0ja@ut.ee
}

Received September 25, 2014; revised December 31, 2014; published online January 15, 2015

\begin{abstract}
The convergence rate of histopolation on an interval with combined splines of class $C^{1}$ having linear/linear rational or quadratic polynomial pieces is studied. The function to histopolate may have finite number of derivative zeros and established convergence rate depends mainly on the behaviour of the derivative near its zeros. Given numerical results are completely consistent with theoretical ones.
\end{abstract}

Keywords: histopolation, rational spline, quadratic spline, convergence rate, comonotone shape-preserving.

AMS Subject Classification: 65D07; 41A15; 41A25.

\section{Introduction}

It is known that the histopolation problem could be reduced to the solution of a corresponding interpolation problem, see, e.g., [14]. Then the derivative of the interpolant serves as histopolant. This idea works in the case of polynomial spline interpolant whose derivative is also a polynomial spline. The convergence rates of polynomial spline interpolants and their derivatives are well known. On the other hand, polynomial spline interpolants and histopolants do not preserve geometric properties like positivity, monotonicity, convexity of the function to approximate. An appropriate tool here is the use of rational or combined splines. But, e.g., the derivative of the quadratic/linear function as a piece of convexity preserving rational spline is not linear/linear function which is a natural piece of monotonicity preserving rational spline. Therefore, the study of histopolation with splines using linear/linear rational pieces is an independent problem. In [3] we established the convergence rate of linear/linear rational spline histopolants in case of strict (uniform) monotonicity. The main purpose of this paper is to find out the convergence rate of histopolating combined splines consisting of linear/linear rational or quadratic polynomial pieces when the function to histopolate is not necessarily monotone. 
While interpolating polynomial splines is a classical research area $[1,15,16]$, there is also extensive literature about shape-preserving spline interpolation, see [6]. Interpolating rational splines of low degree with free parameters as a shape-preserving tool appeared later $[7,8,10]$. An example of the use of interpolating rational splines at the solution of boundary value problems is given in [5]. Histopolation problems with splines are also treated in many works, let us mention of them [16] with several references therein, and $[9,11,12,13]$. In our paper we will follow the main ideas, representation and basic equations from $[2,4]$ for histopolating linear/linear rational or combined splines. The most complicated problem in our research occurs to be the analysis of basic equations determining spline parameters.

\section{Histopolation Problem}

In this section we introduce basic notions and recall some results about the histopolation problem from [4].

Let it be given a mesh $a=x_{0}<x_{1}<\ldots<x_{n}=b$ and real numbers $z_{i}, i=1, \ldots, n$, corresponding to subintervals $\left[x_{i-1}, x_{i}\right]$, i.e. we have a given histogram. We look for a function $S \in C^{1}[a, b]$ which is on each subinterval either a quadratic polynomial or a linear/linear rational function of the form

$$
S(x)=\frac{a_{i}+b_{i}\left(x-x_{i-1}\right)}{1+d_{i}\left(x-x_{i-1}\right)}
$$

with $1+d_{i}\left(x-x_{i-1}\right)>0$ for all $x \in\left[x_{i-1}, x_{i}\right]$ and satisfies the histopolation conditions

$$
\int_{x_{i-1}}^{x_{i}} S(x) d x=z_{i}\left(x_{i}-x_{i-1}\right), \quad i=1, \ldots, n .
$$

In addition, we impose the boundary conditions

$$
S^{\prime}\left(x_{0}\right)=\alpha, \quad S^{\prime}\left(x_{n}\right)=\beta
$$

or

$$
S\left(x_{0}\right)=\alpha, \quad S\left(x_{n}\right)=\beta
$$

for given $\alpha$ and $\beta$. It is known that there are no such a two different combined splines satisfying the same histopolation conditions (2.2) and boundary conditions $(2.3)$ or $(2.4)$, see $[4,8]$.

To determine the kind of subintervals to be rational or quadratic we need the following numbers each of them corresponding to one mesh point

$$
\begin{aligned}
& \delta_{i}=z_{i+1}-z_{i}, \quad i=1, \ldots, n-1 \\
& \delta_{0}=\alpha, \quad \delta_{n}=\beta \text { for boundary conditions }(2.3) \\
& \delta_{0}=z_{1}-\alpha, \quad \delta_{n}=\beta-z_{n} \quad \text { for boundary conditions }(2.4)
\end{aligned}
$$

In general, we choose $\left[x_{i-1}, x_{i}\right]$ to be rational if $\delta_{i-1} \delta_{i}>0$ and quadratic otherwise. We call this choice the comonotone shape-preserving strategy. 
We say that a quadratic section, i.e., a maximal sequence of adjacent quadratic intervals $\left[x_{i}, x_{i+1}\right], \ldots,\left[x_{i+k-1}, x_{i+k}\right]$, has a weak alternation of data if the interval $\left[x_{i-1}, x_{i}\right]$ being rational, intervals $\left[x_{i}, x_{i+1}\right], \ldots,\left[x_{i+k-1}, x_{i+k}\right]$ quadratic and $\left[x_{i-k}, x_{i+k+1}\right]$ again rational then,

$$
\delta_{i}>0, \delta_{i+1} \leqslant 0, \ldots,(-1)^{k} \delta_{i+k-1} \leqslant 0,(-1)^{k} \delta_{i+k}>0
$$

or

$$
\delta_{i}<0, \delta_{i+1} \geqslant 0, \ldots,(-1)^{k} \delta_{i+k-1} \geqslant 0,(-1)^{k} \delta_{i+k}<0 .
$$

In the case of weak alternation of data a solution of the histopolation problem exists (and is unique as was already mentioned). The histopolation problem with the choice of subintervals according to the comonotone shape-preserving strategy and without the weak alternation of data may not have a solution. Because of that we consider the modified comonotone shape-preserving strategy where in absence of the weak alternation of data one of the intervals $\left[x_{i}, x_{i+1}\right]$ or $\left[x_{i+k-1}, x_{i+k}\right]$ is taken to be rational. Then the histopolation problem has always a solution.

\section{Representation and Basic Equations}

In the study of spline algorithms the representation plays always an important role, especially, in nonlinear case. We chose the representation via the derivative values $m_{i}=S^{\prime}\left(x_{i}\right), i=0, \ldots, n$, and histogram heights $z_{i}, i=1, \ldots, n$. Then, with the help of $(2.2),(2.1)$ on $\left[x_{i-1}, x_{i}\right]$ transforms to

$$
\begin{aligned}
S(x)= & z_{i}+h_{i} \frac{m_{i-1}}{\left(\left(\frac{m_{i-1}}{m_{i}}\right)^{1 / 2}-1\right)^{2}} \log \left(\frac{m_{i-1}}{m_{i}}\right)^{1 / 2} \\
& -h_{i} \frac{m_{i-1}}{\left(\left(\frac{m_{i-1}}{m_{i}}\right)^{1 / 2}-1\right)\left(1+t\left(\left(\frac{m_{i-1}}{m_{i}}\right)^{1 / 2}-1\right)\right)}
\end{aligned}
$$

with $x=x_{i-1}+t h_{i}, h_{i}=x_{i}-x_{i-1}$, and on a quadratic interval $\left[x_{i-1}, x_{i}\right]$ we have

$$
S(x)=z_{i}+\frac{h_{i}}{6}\left(\left(-2+6 t-3 t^{2}\right) m_{i-1}+\left(-1+3 t^{2}\right) m_{i}\right) .
$$

We will use the function

$$
\varphi(x)= \begin{cases}\frac{x^{2}(\log x-1)+x}{(x-1)^{2}} & \text { for } x>0, x \neq 0, \\ \frac{1}{2} & \text { for } x=1 .\end{cases}
$$

From the properties of $\varphi$ we need the following

$$
\begin{aligned}
& \varphi(x)>0, \quad \varphi^{\prime}(x)>0, \quad \varphi^{\prime \prime}(x)<0 \quad \text { for } x>0, \\
& \lim _{x \rightarrow 0_{+}} \frac{\varphi(x)}{x}=1, \quad \lim _{x \rightarrow \infty} \frac{\varphi(x)}{\log x}=1, \quad \varphi^{\prime}(1)=\frac{1}{3}, \quad \varphi^{\prime \prime}(1)=-\frac{1}{6} .
\end{aligned}
$$


The continuity of $S$ at $x_{i}$ between rational intervals $\left[x_{i-1}, x_{i}\right]$ and $\left[x_{i}, x_{i+1}\right]$ gives the equation

$$
m_{i}\left(h_{i} \varphi\left(\left(\frac{m_{i-1}}{m_{i}}\right)^{1 / 2}\right)+h_{i+1} \varphi\left(\left(\frac{m_{i+1}}{m_{i}}\right)^{1 / 2}\right)\right)=\delta_{i},
$$

between quadratic intervals $\left[x_{i-1}, x_{i}\right]$ and $\left[x_{i}, x_{i+1}\right]$

$$
\frac{h_{i}}{6} m_{i-1}+\frac{h_{i}+h_{i+1}}{3} m_{i}+\frac{h_{i+1}}{6} m_{i+1}=\delta_{i},
$$

between rational $\left[x_{i-1}, x_{i}\right]$ and quadratic $\left[x_{i}, x_{i+1}\right]$

$$
m_{i}\left(h_{i} \varphi\left(\left(\frac{m_{i-1}}{m_{i}}\right)^{1 / 2}\right)+\frac{h_{i+1}}{3}\right)+\frac{h_{i+1}}{6} m_{i+1}=\delta_{i}
$$

and symmetrical counterpart of equation (3.5) for quadratic $\left[x_{i-1}, x_{i}\right]$ and rational $\left[x_{i}, x_{i+1}\right]$. The boundary conditions (2.3) fix the values $m_{0}=\alpha$ and $m_{n}=\beta$, but (2.4) gives according to (3.1) or (3.2) two additional equations containing $m_{0}, m_{1}$ or $m_{n-1}, m_{n}$, respectively.

In the study of convergence we consider a given sufficiently smooth function $f:[a, b] \rightarrow \mathbb{R}$ and calculate

$$
z_{i}=\frac{1}{h_{i}} \int_{x_{i-1}}^{x_{i}} f(x) d x, \quad i=1, \ldots, n .
$$

The conditions (2.3) and (2.4) are posed in the form

$$
S^{\prime}\left(x_{0}\right)=f^{\prime}\left(x_{0}\right), \quad S^{\prime}\left(x_{n}\right)=f^{\prime}\left(x_{n}\right)
$$

and

$$
S\left(x_{0}\right)=f\left(x_{0}\right), \quad S\left(x_{n}\right)=f\left(x_{n}\right) .
$$

We are interested in the convergence rate of $S-f$ and $S^{\prime}-f^{\prime}$ in uniform norm on $[a, b]$ as $h=\max _{1 \leqslant i \leqslant n} h_{i} \rightarrow 0$.

\section{Analysis of Basic Equations}

Suppose that the given function $f$ has a finite number of points $c_{i}$ in $[a, b]$ such that $f^{\prime}\left(c_{i}\right)=0$. Then the technique of [3] cannot be applied directly. At each point $c_{i}$ we may allow the Taylor expansion

$$
\begin{aligned}
f(x)= & f\left(c_{i}\right)+f^{\prime}\left(c_{i}\right)\left(x-c_{i}\right)+\ldots+\frac{f^{\left(k_{i}-1\right)}\left(c_{i}\right)}{\left(k_{i}-1\right) !}\left(x-c_{i}\right)^{k_{i}-1} \\
& +\frac{f^{\left(k_{i}\right)}\left(c_{i}\right)}{k_{i} !}\left(x-c_{i}\right)^{k_{i}}+o\left(\left(x-c_{i}\right)^{k_{i}}\right)
\end{aligned}
$$

with $f^{\prime}\left(c_{i}\right)=0, \ldots, f^{\left(k_{i}-1\right)}\left(c_{i}\right)=0, f^{\left(k_{i}\right)}\left(c_{i}\right) \neq 0$. Generalizing the situation, we carry out our analysis for the differentiable functions $f$ such that 
$\lim _{x \rightarrow c_{i}, x>c_{i}} f^{\prime}(x) /\left|x-c_{i}\right|^{\alpha_{1}}=\gamma_{1} \neq 0$ and $\lim _{x \rightarrow c_{i}, x<c_{i}} f^{\prime}(x) /\left|x-c_{i}\right|^{\alpha_{2}}=$ $\gamma_{2} \neq 0$ with some constants $\alpha_{1}, \alpha_{2}$ being positive and $\gamma_{1}, \gamma_{2}$. Our reasoning is adequate in the small neighbourhood of points $c_{i}$. For the regions far enough from $c_{i}$ the analysis of [3] could be applied.

Let us indicate some general observations.

Supposing that $f^{\prime} \in \operatorname{Lip} \alpha, \quad 0<\alpha \leqslant 1$, i.e. $\left|f^{\prime}(x)-f^{\prime}(y)\right| \leqslant L|x-y|^{\alpha}$ for some $L$, we have in the Taylor expansion $f(x)=f(a)+f^{\prime}(a)(x-a)+R$ that $|R| \leqslant(L /(1+\alpha))|x-a|^{1+\alpha}$. Then

$$
\delta_{i}=\frac{1}{h_{i+1}} \int_{x_{i}}^{x_{i+1}} f(x) d x-\frac{1}{h_{i}} \int_{x_{i-1}}^{x_{i}} f(x) d x=\frac{1}{2}\left(h_{i}+h_{i+1}\right) f^{\prime}\left(x_{i}\right)+R_{i}
$$

with $\left|R_{i}\right| \leqslant(L /((1+\alpha)(2+\alpha)))\left(h_{i}^{1+\alpha}+h_{i+1}^{1+\alpha}\right)$.

At histopolation always the estimate $\left\|S^{\prime}-f^{\prime}\right\|_{\infty}=O\left(h^{\alpha}\right)$ yields $\|S-f\|_{\infty}=$ $O\left(h^{1+\alpha}\right)$, see [3]. We also discuss in Section 5 how the estimate $\left|m_{i}-f_{i}^{\prime}\right|=$ $O\left(h^{\alpha}\right)$ gives $\left\|S^{\prime}-f^{\prime}\right\|_{\infty}=O\left(h^{\alpha}\right)$.

We will perform the reasoning in particular cases and see later the ways of generalization. For simplicity of presentation, take the uniform partition of $[a, b]$, i.e., $x_{i}=a+i h, \quad i=0, \ldots, n, h=(b-a) / n$. In the case of sufficiently smooth function $f$ we have

$$
\frac{1}{h} \delta_{i}=f_{i}^{\prime}+\frac{h^{2}}{12} f_{i}^{\prime \prime \prime}+O\left(h^{3}\right)
$$

here and in the sequel we mean $f_{i}^{\prime}=f^{\prime}\left(x_{i}\right)$ with similar significance for other functions. Observe that outside of certain (small) neighbourhood of each $c_{i}$ the reasoning of [3] is applicable and the estimate $\left|m_{i}-f_{i}^{\prime}\right|=O\left(h^{2}\right)$ for smooth functions holds (or $\left|m_{i}-f_{i}^{\prime}\right|=O\left(h^{\alpha}\right), 0<\alpha<2$, in the case of lower smoothness, see [3]). Thus, the study only in the neighbourhood of $c_{i}$ is needed. Nevertheless, we give a complete analysis independent of [3].

Let us start with the function $f(x)=x^{2} \operatorname{sgn} x, x \in[-1,1]$.

For $n$ even, we have $x_{i}=0, i=n / 2$, and elementary calculations give

$$
\delta_{i}=\frac{2}{3} h^{2}, \quad \delta_{i+k}=\delta_{i-k}=2 k h^{2}, \quad k=1, \ldots, \frac{n}{2}-1 .
$$

As boundary conditions (3.6) are consistent we choose all subintervals to be rational. For $n$ odd, the point 0 is the midpoint of the interval $\left[x_{i}, x_{i+1}\right]$ with $i=(n-1) / 2$. Then

$$
\delta_{i}=\delta_{i+1}=\frac{13}{12} h^{2}, \quad \delta_{i+k}=(2 k-1) h^{2}, \quad k=2, \ldots,(n-1) / 2,
$$

and

$$
\delta_{i-1}=\delta_{i+k+1}=(2 k+1) h^{2}, \quad k=1, \ldots,(n-3) / 2 .
$$

Here also all subintervals are rational. We will focus our attention mainly to the case of $n$ even.

The equations of the type (3.3) corresponding to the points $x_{j}$ are now

$$
m_{j}\left(\varphi\left(\left(\frac{m_{j-1}}{m_{j}}\right)^{1 / 2}\right)+\varphi\left(\left(\frac{m_{j+1}}{m_{j}}\right)^{1 / 2}\right)\right)=\frac{1}{h} \delta_{j}=f_{j}^{\prime}
$$


and thus

$$
m_{j}-f_{j}^{\prime}=f_{j}^{\prime} \frac{1-\varphi\left(\left(\frac{m_{j-1}}{m_{j}}\right)^{1 / 2}\right)-\varphi\left(\left(\frac{m_{j+1}}{m_{j}}\right)^{1 / 2}\right)}{\varphi\left(\left(\frac{m_{j-1}}{m_{j}}\right)^{1 / 2}\right)+\varphi\left(\left(\frac{m_{j+1}}{m_{j}}\right)^{1 / 2}\right)} .
$$

Denote $\mu_{j}=m_{j-1} / m_{j}, j=1, \ldots, i$, and $\mu_{j}=m_{j} / m_{j-1}, j=i+1, \ldots, n$. Our aim is to estimate the values $\mu_{j}$ and then use them in (4.2). Let us consider the situation at right hand side from $x_{i}=0$ (by symmetry, $\mu_{i+k}=\mu_{i-k+1}$ for all $k$ ), then the equations (4.1) for $j=i+k$ and $j+1$ give

$$
\mu_{j+1}=\frac{\delta_{j+1}}{\delta_{j}} \frac{\varphi\left(\mu_{j}^{-1 / 2}\right)+\varphi\left(\mu_{j+1}^{1 / 2}\right)}{\varphi\left(\mu_{j+1}^{-1 / 2}\right)+\varphi\left(\mu_{j+2}^{1 / 2}\right)}
$$

with $\delta_{j+1} / \delta_{j}=1+1 / k$. Denote $\mu=\left(\mu_{1}, \ldots, \mu_{n}\right)$ and introduce the functions

$$
\Phi_{j}(\mu)=\frac{\varphi\left(\mu_{j-1}^{-1 / 2}\right)+\varphi\left(\mu_{j}^{1 / 2}\right)}{\varphi\left(\mu_{j}^{-1 / 2}\right)+\varphi\left(\mu_{j+1}^{1 / 2}\right)}, \quad \Psi_{j}(\mu)=\frac{\delta_{j}}{\delta_{j-1}} \Phi_{j}(\mu) .
$$

Then the equations (4.3) are $\mu_{j}=\Psi_{j}(\mu)$. We need the following technical

Lemma. Suppose $\mu_{j+l} \in[1+1 /(k+l-1)-\delta, 1+1 /(k+l-1)+\delta], l=0,1,2$, with sufficiently small $\delta>0$. Then for some positive constants $c_{1}, c_{2}$ it holds

$$
\Phi_{j+1}(\mu) \in\left[1-\frac{c_{1}}{k^{3}}-\frac{2}{3} \delta-c_{2} \delta^{2}, 1+\frac{c_{1}}{k^{3}}+\frac{2}{3} \delta+c_{2} \delta^{2}\right] .
$$

Proof. Take $\bar{\mu}_{j+l}=1+1 /(k+l-1), l=0,1,2$, and estimate then $\Phi_{j+1}(\bar{\mu})$. The use of the Taylor expansion

$$
\begin{aligned}
\varphi\left(\bar{\mu}_{j+1}^{1 / 2}\right)= & \varphi\left(\left(1+\frac{1}{k}\right)^{1 / 2}\right)=\varphi(1)+\varphi^{\prime}(1)\left(\left(1+\frac{1}{k}\right)^{1 / 2}-1\right) \\
& +\frac{\varphi^{\prime \prime}(1)}{2}\left(\left(1+\frac{1}{k}\right)^{1 / 2}-1\right)^{2}+\frac{\varphi^{\prime \prime \prime}(\xi)}{6}\left(\left(1+\frac{1}{k}\right)^{1 / 2}-1\right)^{3}
\end{aligned}
$$

and inside that

$$
x^{1 / 2}-1=\frac{1}{2}(x-1)-\frac{1}{8}(x-1)^{2}+O\left((x-1)^{3}\right)
$$

gives

$$
\varphi\left(\bar{\mu}_{j+1}^{1 / 2}\right)=\frac{1}{2}+\frac{1}{6 k}-\frac{1}{16 k^{2}}+O\left(\frac{1}{k^{3}}\right) .
$$

We get also

$$
\varphi\left(\bar{\mu}_{j+1}^{-1 / 2}\right)=\varphi\left(\left(1-\frac{1}{k+1}\right)^{1 / 2}\right)=\frac{1}{2}-\frac{1}{6(k+1)}-\frac{1}{16(k+1)^{2}}+O\left(\frac{1}{k^{3}}\right) .
$$

Then

$$
\varphi\left(\bar{\mu}_{j}^{-1 / 2}\right)+\varphi\left(\bar{\mu}_{j+1}^{1 / 2}\right)=1-\frac{1}{8 k^{2}}+O\left(\frac{1}{k^{3}}\right)
$$


and

$$
\varphi\left(\bar{\mu}_{j+1}^{-1 / 2}\right)+\varphi\left(\bar{\mu}_{j+2}^{1 / 2}\right)=1-\frac{1}{8(k+1)^{2}}+O\left(\frac{1}{k^{3}}\right) .
$$

Now

$$
\Phi_{j+1}(\bar{\mu})=\left(1-\frac{1}{8 k^{2}}+O\left(\frac{1}{k^{3}}\right)\right)\left(1+\frac{1}{8(k+1)^{2}}+O\left(\frac{1}{k^{3}}\right)\right)=1+O\left(\frac{1}{k^{3}}\right) .
$$

If the components of $\mu$ are such that $\mu_{j+l} \in\left[\bar{\mu}_{j+l}-\delta, \bar{\mu}_{j+l}+\delta\right], l=0,1,2$, we take into account $\varphi^{\prime}(1)=1 / 3$, the boundedness of $\varphi^{\prime \prime}$ and the Taylor expansion (4.4) to arrive at the inclusion of $\Phi_{j+1}(\mu)$ stated in the assertion.

As a consequence, in the assumption of Lemma, we get the inclusion

$$
\Psi_{j+1}(\mu) \in\left[1+\frac{1}{k}-\frac{c_{1}}{k^{3}}-\frac{2}{3} \delta-c_{2} \delta^{2}, 1+\frac{1}{k}+\frac{c_{1}}{k^{3}}+\frac{2}{3} \delta+c_{2} \delta^{2}\right],
$$

where the constants $c_{1}, c_{2}$ may be different compared to those of Lemma.

Take in Lemma $\delta=c_{0} / k, c_{0}>0$, then $\Psi_{j+1}(\mu) \in[1+1 / k-\delta, 1+1 / k+\delta]$ if $c_{1} / k^{2}+c_{2} c_{0}^{2} / k \leqslant c_{0} / 3$ for $c_{1}, c_{2}$ in (4.5). This holds for some $c_{0}>0$ and for $k \geqslant k_{0}$ with some fixed $k_{0}$ taken after the choice of $c_{0}$. Basing on the proof of Lemma we get for the solution of $\mu_{j+1}=\Psi_{j+1}(\mu)$ the estimate

$$
\frac{1-\varphi\left(\mu_{j}^{-1 / 2}\right)-\varphi\left(\mu_{j+1}^{1 / 2}\right)}{\varphi\left(\mu_{j}^{-1 / 2}\right)+\varphi\left(\mu_{j+1}^{1 / 2}\right)}=O\left(\frac{1}{k^{2}}\right)+O(\delta)
$$

and, taking into account $f_{j}^{\prime}=2 k h$,

$$
m_{j}-f_{j}^{\prime}=2 k h\left(O\left(\frac{1}{k^{2}}\right)+O(\delta)\right)=O(h) .
$$

It remains to study the behaviour of $m_{j}-f_{j}^{\prime}$ for $j=i, \ldots, i+k_{0}-1$. Because of $f_{j}^{\prime}=2 k h, j=i+k$, it is sufficient to establish the boundedness of $\mu_{j}$ from below and from above by positive constants (not depending on $h$ ) for finite number of indices $k$. The symmetry considerations allow to assert that $m_{i-1}=m_{i+1}$ (still for $n$ even and $i=n / 2$ ) and then $\mu_{i}=\mu_{i+1}$. The equation (4.3) is in this case

$$
\mu_{i}=3 \frac{\varphi\left(\mu_{i}^{-1 / 2}\right)+\varphi\left(\mu_{i}^{1 / 2}\right)}{\varphi\left(\mu_{i}^{-1 / 2}\right)+\varphi\left(\mu_{i+2}^{1 / 2}\right)} .
$$

First, assume that $\mu_{i} \rightarrow \infty$. Then $\varphi\left(\mu_{i}^{1 / 2}\right) \sim\left(\log \mu_{i}\right) / 2, \mu_{i}^{-1} \rightarrow 0$ and $\varphi\left(\mu_{i}^{-1 / 2}\right) \sim \mu_{i}^{-1 / 2} \rightarrow 0$ (here and in the sequel $\sim$ means that the quotient of these terms converges to some positive constant, mainly to 1 ). In the equation (4.7) in the form

$$
\mu_{i}\left(\varphi\left(\mu_{i}^{-1 / 2}\right)+\varphi\left(\mu_{i+2}^{1 / 2}\right)\right)=3\left(\varphi\left(\mu_{i}^{-1 / 2}\right)+\varphi\left(\mu_{i}^{1 / 2}\right)\right)
$$


the right hand side of $(4.8)$ behaves as $3\left(\log \mu_{i}\right) / 2$ but the left hand side increases at least as $\mu_{i}^{1 / 2}$ which gives a contradiction. Secondly, let $\mu_{i} \rightarrow 0$. Then $\varphi\left(\mu_{i}^{1 / 2}\right) \rightarrow 0, \mu_{i}^{-1 / 2} \rightarrow \infty$ and the right hand side of (4.8) is of order $3\left(\log \mu_{i}^{-1}\right) / 2$ while in the left hand side this order has to have the term $\mu_{i} \varphi\left(\mu_{i+2}^{1 / 2}\right)$. This yields that $\mu_{i+2} \rightarrow \infty$ and $\varphi\left(\mu_{i}^{-1 / 2}\right) / \varphi\left(\mu_{i+2}^{1 / 2}\right) \sim 2 \mu_{i} / 3 \rightarrow 0$. The particular case of (4.3) for $j=i+1$ gives

$$
\mu_{i+2}\left(\varphi\left(\mu_{i+2}^{-1 / 2}\right)+\varphi\left(\mu_{i+3}^{1 / 2}\right)\right)=2\left(\varphi\left(\mu_{i}^{-1 / 2}\right)+\varphi\left(\mu_{i+2}^{1 / 2}\right)\right)
$$

where at right the main term is of order $\log \mu_{i+2}$ but at left we have at least $\mu_{i+2}^{1 / 2}$ which is impossible.

This completes the proof of boundedness of $\mu_{i}$ (and $\mu_{i+1}$ ). It remains to carry out the induction step which differs from the just presented reasoning only by details and we omit it.

We have proved the estimate $m_{i}-f_{i}^{\prime}=O(h)$ for the investigated function $f(x)=x^{2} \operatorname{sgn} x, x \in[-1,1]$.

Let us consider now briefly some other particular cases.

The function $f(x)=x^{3}, x \in[-1,1]$, generates $\delta_{j}>0$ for all $j$ and any partition. On uniform partition, for $n$ even and $i=n / 2$ we get $\delta_{i} / h=h^{2} / 2$ and $\delta_{i+k} / h=h^{2}\left(6 k^{2}+1\right) / 2, k \geqslant 1$, and then

$$
\frac{\delta_{i+k}}{\delta_{i+k-1}}=1+\frac{2}{k}+O\left(\frac{1}{k^{2}}\right) .
$$

For $n$ odd and $i=(n-1) / 2$ we have $x_{i}=-h / 2, x_{i+1}=h / 2, \delta_{i} / h=\delta_{i+1} / h$ $=5 h^{2} / 4, \delta_{i+k+1} / h=\left(3(2 k+1)^{2} / 4+1 / 2\right) h^{2}$ and still (4.9) holds. The assertion of Lemma about the inclusion of the value $\Phi_{j+1}(\mu)$ takes places if we choose $\mu_{j+l} \in[1+2 /(k+l-1)-\delta, 1+2 /(k+l-1)+\delta], \quad l=0,1,2$, we only have to take $\bar{\mu}_{j+l}=1+2 /(k+l-1)$ in the proof. Then

$$
\varphi\left(\bar{\mu}_{j}^{-1 / 2}\right)+\varphi\left(\bar{\mu}_{j+1}^{1 / 2}\right)=1-\frac{1}{6 k^{2}}+O\left(\frac{1}{k^{2}}\right)
$$

and other changes in the proof of Lemma are obvious. Taking into account (4.9) we establish the inclusion $\Psi_{j+1}(\mu) \in[1+2 / k-\delta, 1+2 / k+\delta]$ if the inequality

$$
\left(1+\frac{c_{1}}{k^{3}}+\frac{2}{3} \delta+c_{2} \delta^{2}\right)\left(1+\frac{2}{k}+\frac{c_{3}}{k^{2}}\right) \leqslant 1+\frac{2}{k}+\delta
$$

holds $\left(c_{3}\right.$ reflects $\left.(4.9)\right)$. This is achieved with $\delta=c_{0} / k^{2}$ where $c_{0}$ is sufficiently great and $k \geqslant k_{0}$ for some fixed $k_{0}$. Now $\delta_{j} / h=3 k^{2} h^{2}+h^{2} f_{j}^{\prime \prime \prime} / 12$ and instead of (4.2) we have

$$
\begin{aligned}
m_{j}-f_{j}^{\prime}= & f_{j}^{\prime} \frac{1-\varphi\left(\left(\frac{m_{j-1}}{m_{j}}\right)^{1 / 2}\right)-\varphi\left(\left(\frac{m_{j+1}}{m_{j}}\right)^{1 / 2}\right)}{\varphi\left(\left(\frac{m_{j-1}}{m_{j}}\right)^{1 / 2}\right)+\varphi\left(\left(\frac{m_{j+1}}{m_{j}}\right)^{1 / 2}\right)} \\
& +\frac{\frac{h^{2}}{12} f_{j}^{\prime \prime \prime}}{\varphi\left(\left(\frac{m_{j-1}}{m_{j}}\right)^{1 / 2}\right)+\varphi\left(\left(\frac{m_{j+1}}{m_{j}}\right)^{1 / 2}\right)},
\end{aligned}
$$


thus (4.6) is replaced by

$$
m_{j}-f_{j}^{\prime}=3 k^{2} h^{2}\left(O\left(\frac{1}{k^{2}}\right)+O(\delta)\right)+O\left(h^{2}\right)=O\left(h^{2}\right) .
$$

The boundedness of $\mu_{j}$ for finite number of $j$ could be obtained exactly as presented above and, consequently, we have $m_{j}-f_{j}^{\prime}=O\left(h^{2}\right)$ for the function $f(x)=x^{3}, x \in[-1,1]$.

Next, consider the function $f(x)=x^{2}, x \in[-1,1]$, and uniform partition on $[-1,1]$. For $n$ even, we have $x_{i}=0, i=n / 2$, and $z_{i}=z_{i+1}$, thus $\delta_{i}=0$. We have also $\delta_{j}<0, j=0, \ldots, i-1$, and $\delta_{j}>0, j=i+1, \ldots, n$. By the comonotone strategy it should be chosen $\left[x_{i-1}, x_{i}\right]$ and $\left[x_{i}, x_{i+1}\right]$ quadratic, all other particular intervals rational. But there is no weak alternation of data in this section of quadratic intervals and, by the modified strategy, we choose $\left[x_{i-1}, x_{i}\right]$ to be rational, too. For $n$ odd, the point 0 is the midpoint of the interval $\left[x_{i}, x_{i+1}\right]$ with $i=(n-1) / 2$. Then $\delta_{j}<0, j=0, \ldots, i$, and $\delta_{j}>0$, $j=i+1, \ldots, n$. The comonotone strategy makes the subinterval $\left[x_{i}, x_{i+1}\right]$ to be quadratic and all others rational. We have here the weak alternation of data. It is clear that only a finite number of basic equations corresponding to the neighbourhood of $\left[x_{i}, x_{i+1}\right]$ need the study. The equations of the types (3.3)-(3.5) corresponding to the points $x_{i-1}, x_{i}, x_{i+1}$ are now

$$
\begin{gathered}
m_{i-1}\left(\varphi\left(\left(\frac{m_{i-2}}{m_{i-1}}\right)^{1 / 2}\right)+\varphi\left(\left(\frac{m_{i}}{m_{i-1}}\right)^{1 / 2}\right)\right)=\frac{1}{h} \delta_{i-1}=f_{i-1}^{\prime}, \\
m_{i} \varphi\left(\left(\frac{m_{i-1}}{m_{i}}\right)^{1 / 2}\right)+\frac{1}{3} m_{i}+\frac{1}{6} m_{i+1}=\frac{1}{h} \delta_{i}=f_{i}^{\prime}, \\
\frac{1}{6} m_{i}+\frac{1}{3} m_{i+1}+m_{i+1} \varphi\left(\left(\frac{m_{i+2}}{m_{i+1}}\right)^{1 / 2}\right)=\frac{1}{h} \delta_{i+1}=f_{i+1}^{\prime} .
\end{gathered}
$$

Actually, we have to estimate $m_{j}-f_{j}^{\prime}$ for $j=i-1, i, i+1$, for $j<i-1$ and $j>i+1$ the reasoning about the function $f(x)=x^{2} \operatorname{sgn} x$ is valid provided we prove the boundedness of $\mu_{i}=m_{i-1} / m_{i}$ and $\mu_{i+2}=m_{i+2} / m_{i+1}$. We see that, for $n$ even, in (4.11) $f_{i-1}^{\prime}=-2 h$, in (4.12) $f_{i}^{\prime}=0$, in (4.13) $f_{i+1}^{\prime}=2 h$, for $n$ odd, $f_{i-1}^{\prime}=-3 h, f_{i}=-h, f_{i+1}=h$.

Suppose $m_{i-1} / m_{i} \rightarrow \infty$. First, consider the case $c_{1} \leqslant m_{i} / h \leqslant c_{2}$ with some $c_{1}, c_{2}<0$. Dividing (4.12) by $h$, we see that $m_{i+1} / h \rightarrow \infty$. Then (4.13) divided by $h$ gives a contradiction. Secondly, let $m_{i} / h \rightarrow-\infty$. Dividing (4.12) by $m_{i}$ we conclude that $m_{i+1} / m_{i} \rightarrow-\infty$. Then (4.13) divided by $m_{i}$ leads to a contradiction. Thirdly, let $m_{i} / h \rightarrow 0$, however $m_{i}<0$. Then $h / m_{i} \rightarrow-\infty$ and $\left|h / m_{i}\right| \rightarrow \infty$. Consider the case $n$ even (then $f_{i}^{\prime}=0$ ). Dividing (4.12) by $m_{i}$ gives $m_{i+1} / m_{i} \rightarrow-\infty$, we have also $\varphi\left(\left(m_{i-1} / m_{i}\right)^{1 / 2}\right) \sim\left|m_{i+1} / m_{i}\right| / 6$ or

$$
\frac{m_{i-1}}{m_{i}} \sim e^{\frac{1}{3}\left|\frac{m_{i+1}}{m_{i}}\right|} .
$$

Dividing (4.11) by $m_{i}$ we get

$$
\left(\frac{m_{i-1}}{m_{i}}\right)^{1 / 2}+\frac{m_{i-1}}{m_{i}} \varphi\left(\left(\frac{m_{i-2}}{m_{i-1}}\right)^{1 / 2}\right) \sim 2\left|\frac{h}{m_{i}}\right|
$$


and according to (4.14)

$$
e^{\frac{1}{6}\left|\frac{m_{i+1}}{m_{i}}\right|}+e^{\frac{1}{3}\left|\frac{m_{i+1}}{m_{i}}\right|} \varphi\left(\left(\frac{m_{i-2}}{m_{i-1}}\right)^{1 / 2}\right) \sim 2\left|\frac{h}{m_{i}}\right| .
$$

Dividing (4.13) by $m_{i}$ gives

$$
\frac{1}{3}\left|\frac{m_{i+1}}{m_{i}}\right|+\left|\frac{m_{i+1}}{m_{i}}\right| \varphi\left(\left(\frac{m_{i+2}}{m_{i+1}}\right)^{1 / 2}\right) \sim 2\left|\frac{h}{m_{i}}\right| .
$$

The boundedness of $m_{i+2} / m_{i+1}$ means that $\left|m_{i+1} / m_{i}\right|$ and $2\left|h / m_{i}\right|$ have the same order and the left hand side of (4.15) has also the order of $\left|m_{i+1} / m_{i}\right|$ which is impossible. It remains to consider the case $m_{i+2} / m_{i+1} \rightarrow \infty$. Dividing the counterpart of (4.11) corresponding to $x_{i+2}$ by $m_{i+1}$ gives

$$
\frac{m_{i+2}}{m_{i+1}}\left(\varphi\left(\left(\frac{m_{i+1}}{m_{i+2}}\right)^{1 / 2}\right)+\varphi\left(\left(\frac{m_{i+3}}{m_{i+2}}\right)^{1 / 2}\right)\right) \sim \frac{h}{m_{i+1}},
$$

which means also that $h / m_{i+1} \rightarrow \infty$ because $m_{i+1} / m_{i+2} \rightarrow 0$. Then (4.13) divided by $m_{i+1}$ implies that $h / m_{i+1}$ has the order of $\log \left(m_{i+2} / m_{i+1}\right)$ which is in contradiction with (4.16). Consider now the case of $n$ odd. Dividing (4.12) by $m_{i}$ we get

$$
\varphi\left(\left(\frac{m_{i-1}}{m_{i}}\right)^{1 / 2}\right)+\frac{1}{3}=\frac{1}{6}\left|\frac{m_{i+1}}{m_{i}}\right|+\left|\frac{h}{m_{i}}\right| .
$$

The possibility $\varphi\left(\left(m_{i-1} / m_{i}\right)^{1 / 2}\right) \sim\left|m_{i+1} / m_{i}\right|$ (the quotient is bounded from above and below by positive constants) could be treated as in the case of $n$ even. If $\varphi\left(\left(m_{i-1} / m_{i}\right)^{1 / 2}\right) \sim\left|h / m_{i}\right|$ then dividing (4.11) by $m_{i}$ we get

$$
\frac{m_{i-1}}{m_{i}} \varphi\left(\left(\frac{m_{i-2}}{m_{i-1}}\right)^{1 / 2}\right)+\frac{m_{i-1}}{m_{i}} \varphi\left(\left(\frac{m_{i}}{m_{i-1}}\right)^{1 / 2}\right)=3\left|\frac{h}{m_{i}}\right| .
$$

Here the right hand side is of order $\log \left(m_{i-1} / m_{i}\right)$ while the left hand side is of order at least $\left(m_{i-1} / m_{i}\right)^{1 / 2}$ which is a contradiction. This completes the proof of the boundedness of $m_{i-1} / m_{i}$ from above.

Suppose next that $m_{i-1} / m_{i} \rightarrow 0$, then $m_{i} / m_{i-1} \rightarrow \infty$. Dividing (4.11) by $m_{i-1}$ we get

$$
\varphi\left(\left(\frac{m_{i-2}}{m_{i-1}}\right)^{1 / 2}\right)+\varphi\left(\left(\frac{m_{i}}{m_{i-1}}\right)^{1 / 2}\right)=l\left|\frac{h}{m_{i-1}}\right|
$$

with $l=2$ or $l=3$ depending on $n$ to be even or odd. This means that $\left|h / m_{i-1}\right| \rightarrow \infty$. If $\varphi\left(\left(m_{i-2} / m_{i-1}\right)^{1 / 2}\right)$ is of order $\left|h / m_{i-1}\right|$ then

$$
\frac{m_{i}}{m_{i-1}} \sim e^{k\left|\frac{h}{m_{i-1}}\right|}
$$

with some constant $k$ (here also, $\sim$ means that the quotient is two-sided bounded). Dividing (4.12) by $m_{i-1}$ we see that

$$
\frac{m_{i+1}}{m_{i-1}} / \frac{m_{i}}{m_{i-1}} \rightarrow-2
$$


Dividing (4.13) by $m_{i-1}$ gives

$$
\frac{1}{6} \frac{m_{i}}{m_{i-1}}+\left(\frac{1}{3}+\varphi\left(\left(\frac{m_{i+2}}{m_{i+1}}\right)^{1 / 2}\right)\right) \frac{m_{i+2}}{m_{i-1}}=-l\left|\frac{h}{m_{i-1}}\right|
$$

with $l=1$ or $l=2$ and this is a contradiction due to (4.18) and (4.17). If $\varphi\left(\left(m_{i-2} / m_{i-1}\right)^{1 / 2}\right)$ is of order $\left|h / m_{i-1}\right|$ then

$$
\frac{m_{i-2}}{m_{i-1}} \sim e^{k\left|\frac{h}{m_{i-1}}\right|}
$$

Now the counterpart of $(4.11)$ corresponding to the point $x_{i-2}$ divided by $m_{i-1}$ gives

$$
\frac{m_{i-2}}{m_{i-1}} \varphi\left(\left(\frac{m_{i-3}}{m_{i-2}}\right)^{1 / 2}\right)+\left(\frac{m_{i-2}}{m_{i-1}}\right)^{1 / 2} \sim\left|\frac{h}{m_{i-1}}\right|,
$$

which is a contradiction due to (4.19) and this ends the proof of boundedness of $m_{i-1} / m_{i}$ from below by a positive constant.

As any point $x_{j}$ is an endpoint of a rational interval and the quotient of two values $m_{j}$ in any rational interval is bounded from above and below by positive constants, we obtain the estimate $m_{j}-f_{j}^{\prime}=O(h)$ for all $j$ like in the case of function $f(x)=x^{2} \operatorname{sgn} x$.

The function $f(x)=|x|^{3}, x \in[-1,1]$, could be treated joining the arguments from treatments of two previous functions. Here we have the estimate $m_{j}-f_{j}^{\prime}=O\left(h^{2}\right)$.

Ending this section let us indicate the framework at establishing the estimates. First, we prove the boundedness of $\mu_{j}$ for finite number of indices $j=i, \ldots, i+k_{0}$ (or for $j<i$ ), with suitable fixed $k_{0}$ and then use (4.6) or (4.10). For $j>i+k_{0}$ (or $j<i-k_{0}$ ) we use Bohl-Brouwer fixed point principle as it was done by us several times $[2,3,4]$ basing on inclusions (4.5) or its analogue in the analysis of function $f(x)=x^{3}$.

However, we need a boundary value $\mu_{j+1}=\mu_{i+k_{0}}$ but, e.g., we take it from the estimates $m_{j}=f_{j}^{\prime}+\alpha_{1}, m_{j+1}-f_{j+1}^{\prime}=\alpha_{2},\left|\alpha_{1}\right| \leqslant c h,\left|\alpha_{2}\right| \leqslant c h, c=$ const, then $\mu_{j+1}=m_{j+1} / m_{j}=1+1 / k_{0}+\delta,|\delta| \leqslant c_{0} / k_{0}$. Such an estimate is valid due to (4.6) but works as well if we use (4.10). At the other end we choose as boundary condition $\mu_{n+1}=1+2 / n$ or $\mu_{n+1}=1+1 / n$ for the cases $f(x)=x^{2}$ or $f(x)=x^{3}$, respectively.

\section{Convergence Results}

In Section 4 we established the estimates $m_{i}-f_{i}^{\prime}=O\left(h^{\alpha}\right), 0<\alpha \leqslant 2$, depending on the function of $f$ to histopolate. In the beginning we show how this implies the estimate $\left\|S^{\prime}-f^{\prime}\right\|_{\infty}=O\left(h^{\alpha}\right)$. We mentioned already that this yields $\|S-f\|_{\infty}=O\left(h^{1+\alpha}\right)$.

The representation (3.1) gives on a rational interval $\left[x_{i-1}, x_{i}\right]$

$$
S^{\prime}(x)=\frac{m_{i-1}}{\left(1+\frac{x-x_{i-1}}{h}\left(\left(\frac{m_{i-1}}{m_{1}}\right)^{1 / 2}-1\right)\right)^{2}}
$$


and on a quadratic interval $\left[x_{i-1}, x_{i}\right]$ from (3.2) follows

$$
S^{\prime}(x)=(1-t) m_{i-1}+t m_{i},
$$

actually, as the linear interpolation representation for derivative $S^{\prime}$. On rational intervals we have using (5.1) and (4.1)

$$
\begin{aligned}
& S^{\prime}(x)-f^{\prime}(x)=S^{\prime}(x)-f_{j-1}^{\prime}+f_{j-1}^{\prime}-f^{\prime}(x) \\
& =\frac{\frac{1}{h} \delta_{i-1}}{\left(1+\frac{x-x_{i-1}}{h}\left(\left(\frac{m_{i-1}}{m_{i}}\right)^{1 / 2}-1\right)\right)^{2}\left(\varphi\left(\left(\frac{m_{i-2}}{m_{i-1}}\right)^{1 / 2}\right)+\varphi\left(\left(\frac{m_{i}}{m_{i-1}}\right)^{1 / 2}\right)\right)} \\
& \quad-f_{i-1}^{\prime}+O(h) .
\end{aligned}
$$

In the case of function $f(x)=x^{2}$ and $f(x)=x^{2} \operatorname{sgn} x$ it holds $\delta_{i-1} / h=f_{i-1}^{\prime}$ and (5.3) gives $S^{\prime}(x)-f^{\prime}(x)=O(h)$ because $f_{j+1}^{\prime}=2 k h, j=n / 2+k$ (or $j+1=(n-1) / 2+k), j \geqslant n / 2$ and $\mu_{j+1}=1+O(1 / k)$. Near the central knot we only use the boudedness of $\mu_{j}$. The same reasoning works as well in the case of functions $f(x)=x^{3}$ and $f(x)=|x|^{3}$ for $k \leqslant k_{0}, k_{0}$ fixed, because of $f_{j+1}^{\prime}=3 k^{2} h^{2}$ and $f_{i-1}^{\prime}-f(x)=O\left(h^{2}\right)$ due to $f^{\prime \prime}(x)=O(h), 0 \leqslant x \leqslant x_{n / 2+k_{0}}$. Then we obtain $S^{\prime}(x)-f^{\prime}(x)=O\left(h^{2}\right)$. For $k>k_{0}$, use the expansion

$$
\begin{aligned}
S^{\prime}(x)-f^{\prime}(x)= & m_{i-1}-f_{i-1}^{\prime}+\left(x-x_{i-1}\right)\left(S_{i-1}^{\prime \prime}-f_{i-1}^{\prime \prime}\right) \\
& +\frac{\left(x-x_{i-1}\right)^{2}}{2}\left(S^{\prime \prime \prime}-f^{\prime \prime \prime}\right)(\xi) .
\end{aligned}
$$

Let us show how to establish $S_{i-1}^{\prime \prime}-f_{i-1}^{\prime \prime}=O(h)$. From (5.1) follows

$$
S_{i-1}^{\prime \prime}=-\frac{2 m_{i-1}}{h}\left(\left(\frac{m_{i-1}}{m_{i}}\right)^{1 / 2}-1\right) \text {. }
$$

It was shown that $\left(i \geqslant n / 2+k_{0}\right) \mu_{i}=m_{i} / m_{i-1}=1+2 / k+O\left(1 / k^{2}\right)$, then $\left(m_{i-1} / m_{i}\right)^{1 / 2}-1=-1 / k+O\left(1 / k^{2}\right)$ (see, e.g., [3]), $f_{i-1}^{\prime}=3 k^{2} h^{2}$ and we get due to $m_{i-1}=f_{i-1}^{\prime}+O\left(h^{2}\right)$

$$
S_{i-1}^{\prime \prime}=\frac{-2\left(3 k^{2} h^{2}+O\left(h^{2}\right)\right)}{h}\left(-\frac{1}{k}+O\left(\frac{1}{k^{2}}\right)\right)=6 k h+O(h) .
$$

Now, as $f_{i-1}^{\prime \prime}=6 k h$, we get $S_{i-1}^{\prime \prime}-f_{i-1}^{\prime \prime}=O(h)$. In addition, (5.1) gives

$$
S^{\prime \prime \prime}(x)=\frac{6 m_{i-1} \frac{1}{h^{2}}\left(\left(\frac{m_{i-1}}{m_{i}}\right)^{1 / 2}-1\right)^{2}}{\left(1+\frac{x-x_{i-1}}{h}\left(\left(\frac{m_{i-1}}{m_{i}}\right)^{1 / 2}-1\right)\right)^{4}}
$$

and using again $\left(m_{i-1} / m_{i}\right)^{1 / 2}-1=-1 / k+O\left(1 / k^{2}\right), m_{i-1}=3 k^{2} h^{2}+O\left(h^{2}\right)$, we obtain the uniform boundedness of $S^{\prime \prime \prime}$ and from (5.4) $S^{\prime}(x)-f^{\prime}(x)=O\left(h^{2}\right)$ for $k>k_{0}$, too. On the quadratic interval (5.2) gives

$$
S^{\prime}(x)-f^{\prime}(x)=(1-t)\left(m_{i-1}-f_{i-1}^{\prime}\right)+t\left(m_{i}-f_{i}^{\prime}\right)+(1-t) f_{i-1}^{\prime}+t f_{i}^{\prime}-f^{\prime}(x)
$$

and from this the required order follows if we take into account the order of linear interpolation on dependence on the smoothness of $f$. 
It is quite clear how all presented reasonings could be generalized to the case considered at the beginning of Section 4. Let us indicate some important moments. If in the point $c_{i}$ the function $f^{\prime}$ do not change the sign, we should argue as in the case of $f(x)=x^{2} \operatorname{sgn} x$ or $f(x)=x^{3}$. Then in (4.3) $\delta_{j+1} / \delta_{j}=1+$ $l / k+O\left(1 / k^{2}\right)$ with some constant $l$ which can be fractional. Such a fractionality of constants appears also in other formulae but all presented steps of the proofs are valid. If $f^{\prime}$ changes the sign in the point $c_{i}$, we follow the proof of the cases of $f(x)=x^{2}$ or $f(x)=|x|^{3}$ and again the fractionality phenomina should be taken into account. We have proved the following

Theorem. Suppose that a function $f$ has a finite number of points $c_{i}$ in $[a, b]$ such that $f^{\prime}\left(c_{i}\right)=0$ and

$$
\lim _{\substack{x \rightarrow c_{i} \\ x<c_{i}}} \frac{f^{\prime}(x)}{\left|x-c_{i}\right|^{\alpha_{i 1}}}=\gamma_{i 1} \neq 0, \quad \lim _{\substack{x \rightarrow c_{i} \\ x>c_{i}}} \frac{f^{\prime}(x)}{\left|x-c_{i}\right|^{\alpha_{i 2}}}=\gamma_{i 2} \neq 0 .
$$

Let $\alpha=\min _{i} \min \left\{\alpha_{i 1}, \alpha_{i 2}, 2\right\}$. We also assume that $f^{\prime} \in$ Lip $\alpha$ if $0<\alpha \leqslant 1$ or $f^{\prime \prime} \in \operatorname{Lip}(\alpha-1)$ if $1<\alpha \leqslant 2$. Then the combined histopolating spline $S$ which is constructed by comonotone or modified comonotone strategy, has in the uniform norm on $[a, b]$ the convergence rate $\|S-f\|_{\infty}=O\left(h^{1+\alpha}\right)$ together with $\left\|S^{\prime}-f^{\prime}\right\|_{\infty}=O\left(h^{\alpha}\right)$.

Remark. We formulated and proved the results in the case of uniform mesh. It is quite evident that our arguments work also in the case of a mesh with $0<q_{1} \leqslant h_{j} / h_{i} \leqslant q_{2}$ for $|i-j|=1$ where $q_{1}, q_{2}$ are constants. The reason here is that far enough from the points $c_{i}$ the results hold by [3], but in finite number of intervals around the points $c_{i}$ such nonuniformity can change only the constants, not the order of all given estimates. However, around the points $c_{i}$ where the theoretical rate of convergence is lower than in regions with strict uniform monotonicity, we can use the mesh with smaller step as the idea of adaptive meshes. This compensates such a lower rate.

\section{$6 \quad$ Numerical Tests}

We histopolated the functions $f(x)=x^{2} \operatorname{sgn} x, f(x)=x^{3}, f(x)=x^{2}$ and $f(x)=|x|^{3}$ on the interval $[-1,1]$. For the first and third functions we obtain convergence rate $O\left(h^{2}\right)$ and for others the rate $O\left(h^{3}\right)$. In our tests we used the uniform mesh, for the third function we used also a nonuniform mesh, where central knots were calculated as $x_{(n+1) / 2}=h / 2, x_{(n+1) / 2-1}=-h / 2$, $x_{(n+1) / 2-2}=-3 h / 2, x_{(n+1) / 2+1}=3 h / 5$. Other knots were spaced uniformly on rest parts of the interval, i.e., $x_{i}=x_{0}+i\left(x_{(n+1) / 2-2}-x_{0}\right) /((n+1) / 2-2)$, $i=1, \ldots,(n+1) / 2-3$ and $x_{(n+1) / 2+1+i}=x_{n}-(n-1)\left(x_{n}-x_{(n+1) / 2+1}\right) /((n+$ 1) $/ 2-2), i=1, \ldots, n-1$. For the function $f(x)=x^{2}$ we made test for both, $n$ odd and $n$ even. Other functions were tested only for $n$ odd or only for $n$ even and on uniform mesh. Selection of the subinterval was made by comonotone shape-preserving strategy in case of $n$ odd and by modified comonotone shapepreserving strategy in case of $n$ even. 
Table 1. Numerical results for $f(x)=x^{2}, x \in[-1,1]$, uniform mesh, $n$ odd.

\begin{tabular}{llllll}
\hline$n$ & 5 & 15 & 45 & 135 & 405 \\
\hline$\varepsilon_{n}$ & $7.34 \times 10^{-3}$ & $8.39 \times 10^{-4}$ & $9.33 \times 10^{-5}$ & $1.04 \times 10^{-5}$ & $1.15 \times 10^{-6}$ \\
$\varepsilon_{n} / \varepsilon_{n / 3}$ & & 8.741958 & 9.000082 & 9.000000 & 9.000000 \\
\hline
\end{tabular}

Table 2. Numerical results for $f(x)=x^{2}, x \in[-1,1]$, uniform mesh, $n$ even.

\begin{tabular}{llllll}
\hline$n$ & 8 & 16 & 32 & 64 & 128 \\
\hline$\varepsilon_{n}$ & $7.88 \times 10^{-3}$ & $1.97 \times 10^{-3}$ & $4.93 \times 10^{-4}$ & $1.23 \times 10^{-4}$ & $3.08 \times 10^{-5}$ \\
$\varepsilon_{n} / \varepsilon_{n / 2}$ & & 3.998068 & 3.999997 & 4.000000 & 4.000000 \\
\hline
\end{tabular}

Table 3. Numerical results for $f(x)=x^{2}, x \in[-1,1]$, nonuniform mesh, $n$ odd.

\begin{tabular}{llllll}
\hline$n$ & 5 & 15 & 45 & 135 & 405 \\
\hline$\varepsilon_{n}$ & $3.95 \times 10^{-2}$ & $9.44 \times 10^{-4}$ & $1.05 \times 10^{-4}$ & $1.16 \times 10^{-5}$ & $1.29 \times 10^{-6}$ \\
$\varepsilon_{n} / \varepsilon_{n / 3}$ & & 41.790849 & 9.012392 & 9.003344 & 9.001046 \\
\hline
\end{tabular}

Table 4. Numerical results for $f(x)=x^{3}, x \in[-1,1]$, uniform mesh, $n$ even.

\begin{tabular}{llllll}
\hline$n$ & 8 & 16 & 32 & 64 & 128 \\
\hline$\varepsilon_{n}$ & $3.70 \times 10^{-3}$ & $5.23 \times 10^{-4}$ & $6.81 \times 10^{-5}$ & $8.66 \times 10^{-6}$ & $1.09 \times 10^{-6}$ \\
$\varepsilon_{n} / \varepsilon_{n / 2}$ & & 7.086933 & 7.681549 & 7.856177 & 7.931600 \\
\hline
\end{tabular}

Table 5. Numerical results for $f(x)=\left|x^{3}\right|, x \in[-1,1]$, uniform mesh, $n$ odd.

\begin{tabular}{llllll}
\hline$n$ & 5 & 15 & 45 & 135 & 405 \\
\hline$\varepsilon_{n}$ & $1.27 \times 10^{-2}$ & $6.32 \times 10^{-4}$ & $2.47 \times 10^{-5}$ & $9.31 \times 10^{-7}$ & $3.47 \times 10^{-8}$ \\
$\varepsilon_{n} / \varepsilon_{n / 3}$ & & 20.130665 & 25.566869 & 26.557522 & 26.858469 \\
\hline
\end{tabular}

Table 6. Numerical results for $f(x)=x^{2} \operatorname{sgn} x, x \in[-1,1]$, uniform mesh, $n$ even.

\begin{tabular}{llllll}
\hline$n$ & 8 & 16 & 32 & 64 & 128 \\
\hline$\varepsilon_{n}$ & $4.39 \times 10^{-3}$ & $1.10 \times 10^{-3}$ & $2.75 \times 10^{-4}$ & $6.86 \times 10^{-5}$ & $1.72 \times 10^{-5}$ \\
$\varepsilon_{n} / \varepsilon_{n / 2}$ & & 3.998591 & 3.999998 & 4.000000 & 4.000000 \\
\hline
\end{tabular}

We used the boundary conditions $\alpha=f^{\prime}\left(x_{0}\right)$ and $\beta=f^{\prime}\left(x_{n}\right)$. The approximates to the errors $\|S-f\|_{\infty}$ were calculated on ten times refined grid as

$$
\varepsilon_{n}=\max _{1 \leqslant i \leqslant n} \max _{1 \leqslant k \leqslant 10}\left|S\left(x_{i-1}+\frac{k h}{10}\right)-f\left(x_{i-1}+\frac{k h}{10}\right)\right| .
$$


Nonlinear system of $m_{i}, i=0, \ldots, n$, was solved by Newton's method. Results of numerical tests are presented in Tables 1-6.

Numerical results are completely in concordance with theoretical ones.

\section{Acknowledgement}

We wish to thank the referee for the valuable remarks about the preliminary version of the paper.

This work was supported by institutional research funding IUT20-57 of the Estonian Ministry of Education and Research.

\section{References}

[1] C. de Boor. A practical guide to splines. Springer-Verlag, New York, 2001.

[2] M. Fischer and P. Oja. Monotonicity preserving rational spline histopolation. J. Comput. Appl. Math., 175:195-208, 2005.

[3] M. Fischer and P. Oja. Convergence rate of rational spline histopolation. Math. Model. Anal., 12(1):1-10, 2007.

[4] M. Fischer, P. Oja and H. Trossmann. Comonotone shape-preserving spline histopolation. J. Comput. Appl. Math., 200(1):127-139, 2007.

[5] E. Ideon and P. Oja. Linear/linear rational spline collocation for linear boundary value problems. J. Comput. Appl. Math, 263:32-44, 2014. http://dx.doi.org/10.1016/j.cam.2013.11.028.

[6] B.I. Kvasov. Methods of Shape-Preserving Spline Approximation. World Scientific Publishing Co, River Edge, NJ, 2000.

[7] P. Oja. Low degree rational spline interpolation. BIT, 37:900-908, 1997. http://dx.doi.org/10.1007/BF02510359.

[8] P. Oja. Comonotone adaptive interpolating splines. BIT, 42(4):842-855, 2002. http://dx.doi.org/10.1023/A:1021908705602.

[9] M. Sakai and R.A. Usmani. A shape preserving area true approximation of histogram by rational splines. BIT, 28:329-339, 1988.

http://dx.doi.org/10.1007/BF01934096.

[10] R. Schaback. Adaptive rational splines. Constr. Approx., 6:167-179, 1990. http://dx.doi.org/10.1007/BF01889356.

[11] J.W. Schmidt. Constrained smoothing of histograms by quadratic splines. Computing, 48:97-109, 1992. http://dx.doi.org/10.1007/BF02241708.

[12] J.W. Schmidt and W. Heß. Shape preserving $C^{2}$-spline histopolation. J. Approx. Theory, 75:325-345, 1993. http://dx.doi.org/10.1006/jath.1993.1106.

[13] J.W. Schmidt, W. Heß and Th. Nordheim. Shape preserving histopolation using rational quadratic splines. Computing, 44:245-258, 1990. http://dx.doi.org/10.1007/BF02262220.

[14] I.J. Schoenberg. Splines and histograms. Internat. Ser. Numer. Math., 21, 1973.

[15] L.L. Schumaker. Spline functions: basic theory. Robert E. Krieger Publishing Co., Inc., Malabar, FL, 1993.

[16] H. Späth. One Dimensional Spline Interpolation Algorithms. A. K. Peters, Massachusetts, 1995. 